\title{
Analysis of the spectral width and validation of the LHBEAM code
}

\author{
N. Bertelli*, O. Maj ${ }^{\dagger}$, E. Poli ${ }^{\dagger}$, G. V. Pereverzev ${ }^{\dagger}$, Y. Peysson** and \\ J. Decker** \\ *FOM-Institute for Plasma Physics Rijnhuizen, Association EURATOM-FOM, Trilateral Euregio \\ Cluster, Nieuwegein, The Netherlands, www.rijnhuizen.nl \\ ${ }^{\dagger}$ Max-Planck-Institut für Plasmaphysik, IPP, D-85748 Garching, Germany \\ ${ }^{* *}$ Associaton Euratom-CEA, CEA/DSM/DRFC, CEA-Cadarache, 13108, Saint Paul lez Durance, \\ France
}

\begin{abstract}
A crucial point of the theoretical study of lower-hybrid (LH) current drive in a tokamak plasma is the spectral gap problem, i.e., the fact that the parallel (to the magnetic field) refractive index spectrum generated at the plasma edge does not appear to be wide enough for the interaction of the wave with a large number of electrons. This is in contrast with experimental observations. Diffraction is one of the mechanisms that can lead to the observed wave spectrum broadening and solve the spectral gap problem. For this reason, a new beam tracing code, LHBEAM, has been developed in order to study the diffraction effects on the propagation and the absorption of LH waves in tokamak plasma. In this work, the parallel spectral width is addressed on the basis of the beam tracing approximate solution. A preliminary implementation of the results is done in LHBEAM which has been also compared with the ray tracing code $\mathrm{C} 3 \mathrm{PO}$ for the assessment of the trajectory of the central ray and of the evolution of the parallel refractive index on this ray.
\end{abstract}

Keywords: lower hybrid waves, current drive, spectral gap, spectral width, diffraction PACS: $52.35 . \mathrm{Hr}, 52.55 . \mathrm{Wq}$

\section{ON THE SPECTRUM OF THE BEAM TRACING SOLUTION}

With regard to the description of LH wave beams, diffraction is an important aspect for both the broadening of the beam cross-section and the wave energy absorption mechanism [1] which, in particular, involves the parallel (to the magnetic field) energy spectrum [2,3]. The beam tracing description [4] of electromagnetic wave beams allows us to take into account the effects of diffraction. A new LH beam tracing code, LHBEAM [5], has been developed on the basis of the electron-cyclotron code TORBEAM [6], and the first results provide a clear picture of diffraction effects on the beam width. We shall now address the effects of diffraction on the parallel energy spectrum as relevant to the calculation of LH power deposition.

The construction of the beam tracing solution requires the identification of a specific geometric optics ray, the reference ray, that represents the path of the beam in the plasma. On making use of normalized coordinates $\mathbf{x}=\mathbf{r} / L, L$ being the scale length of the plasma inhomogeneity, the reference ray amounts to a curve $\mathbf{x}=\mathbf{x}_{0}(\tau)$ which is computed together with the carried refractive index $\mathbf{N}=\mathbf{N}_{0}(\tau)$. Near the reference ray, we can introduce curvilinear coordinates $(\tau, \mathbf{v}), \mathbf{v}=\left(v^{1}, v^{2}\right)$, with $\mathbf{v}=0$ on the reference ray. In terms of $(\tau, \mathbf{v})$, the beam tracing solution for the electric field of a purely Gaussian beam 
reads $[4,5]$

$$
\mathbf{E}_{\mathrm{BT}}(\tau, \mathbf{v})=\mathbf{E}_{0}(\tau) e^{i k N_{\alpha}(\tau) v^{\alpha}} e^{-\frac{k}{2} A_{\alpha \beta}(\tau) v^{\alpha} v^{\beta}},
$$

where, here and throughout the paper, the sum over repeated indices is implied, $k=$ $\omega L / c \gg 1$ is the normalized frequency of the beam ( $c$ being the speed of light), $\mathbf{E}_{0}(\tau)$ is the wave electric field on the reference ray, $N_{\alpha}(\tau)=\mathbf{x}_{\alpha}(\tau) \cdot \mathbf{N}_{0}(\tau)$ is the component of the refractive index along the direction of the coordinate $v^{\alpha}$, with $\mathbf{x}_{\alpha}(\tau)=\partial \mathbf{x} /\left.\partial v^{\alpha}\right|_{\mathbf{v}=0}$, and the matrix $A(\tau)=\left(A_{\alpha \beta}(\tau)\right)$ is retrieved from the solution of the beam tracing equations given by the codes $[5,6]$. Let us recall that $\mathbf{v}$ can span either a curved surface across the beam or a plane. Usually the latter case is considered, but here we shall need curved cross-sections.

The specific form (1) of the beam tracing solution naturally entails a description of the beam in terms of the evolution in $\tau$ of the beam cross-sections spanned by the transversal coordinates $\mathbf{v}=\left(v^{1}, v^{2}\right)$; it must be noted, however, that the evolving cross-sections, or, equivalently, the coordinates $\mathbf{v}=\left(v^{1}, v^{2}\right)$, are arbitrary.

With this picture in mind, we consider the energy spectrum of the cross-sections only. First, we compute the squared absolute value of the Fourier transform $\widetilde{\mathbf{E}}_{\mathrm{BT}}\left(\tau, N_{\alpha}\right)$ of (1) with respect to $\mathbf{v}$, upon letting $\mathbf{v}$ run over the whole two-dimensional space; the result defines a $\tau$-dependent distribution for the components $N_{\alpha}$ of the refractive index along $v^{\alpha}$, namely,

$$
\left|\widetilde{\mathbf{E}}_{\mathrm{BT}}\left(\tau, N_{\alpha}\right)\right|^{2} \propto\left|\widetilde{\mathbf{E}}_{0}(\tau)\right|^{2} e^{-k B_{1}^{\alpha \beta}(\tau)\left(N_{\alpha}-N_{\alpha}(\tau)\right)\left(N_{\beta}-N_{\beta}(\tau)\right)}
$$

where $B_{1}^{\alpha \beta}(\tau)=\operatorname{Re} B^{\alpha \beta}, B(\tau)=\left(B^{\alpha \beta}(\tau)\right)$ being the inverse matrix of $A(\tau)=\left(A_{\alpha \beta}(\tau)\right)$. Strictly speaking, the domain of $\mathbf{v}$ is limited to a small neighbourhood of the reference ray, rather than covering the whole two-dimensional space, but this technical difficulty can be overcome by an extension argument which allows us to prove that (2) approximates the energy spectrum of the cross-sections within an accuracy of $O(1 / \sqrt{k})$.

The substantial difficulty in the application of (2) to the description of the waveparticle interaction is twofold: first, we need to show that, during their interaction with the wave, particles are sensitive to the cross-sections of the beam only, and not to the whole beam; then, we have to determine which are the relevant cross-sections. The latter point is particularly important since the distribution (2) depends strongly on the choice of the beam cross-sections. It appears that a fully satisfactory and rigorous solution of these two points requires a careful analysis from first principles; here, we limit ourselves to simple heuristic considerations.

For LH waves we expect that the wave-particle interaction depends on the parallel (to the magnetic field) spectrum only. Hence, for the application of (2), we require that the group velocity $\left(\propto d \mathbf{x}_{0}(\tau) / d \tau\right)$ never lines up with the magnetic field unit vector $\mathbf{b}\left(\mathbf{x}_{0}(\tau)\right)$ throughout the propagation, so that the magnetic field keeps tangent to some beam cross-sections that we have now to determine. Since the orbits of the particles, roughly speaking, follow the magnetic field line, it is natural to assume that the magnetic field line passing through $\mathbf{x}_{0}(\tau)$ belongs to the beam cross-section passing through the same point $\mathbf{x}_{0}(\tau)$. There is, however, another degree of freedom that should be fixed in order to determine uniquely the beam cross-sections, and the eventual result will depend on this choice: this is still an open problem; in the following we make the simplest 
choice: we assume that the relevant beam cross-section near the reference ray can be parametrized by $\mathbf{x}(\tau, s, u)=\mathbf{x}(\tau, s)+\mathbf{e}_{u}(\tau) u$, where $\mathbf{x}(\tau, s)$ is the parametrization of the magnetic field line passing through $\mathbf{x}_{0}(\tau)$ in terms of its own arc length $s$, and $\mathbf{e}_{u}(\tau) \propto\left(d \mathbf{x}_{0}(\tau) / d \tau\right) \times \mathbf{b}\left(\mathbf{x}_{0}(\tau)\right)$ is a vector orthogonal to both the group velocity $(\propto$ $\left.d \mathbf{x}_{0}(\tau) / d \tau\right)$ and the magnetic field unit vector $\mathbf{b}\left(\mathbf{x}_{0}(\tau)\right)$. The corresponding coordinates $\mathbf{v}=\left(v^{1}, v^{2}\right)$ can be defined by $v^{1}=s$ and $v^{2}=u$ but this is not mandatory. Then, we find $\mathbf{b}\left(\mathbf{x}_{0}(\tau)\right)=b^{\alpha}(\tau) \mathbf{x}_{\alpha}(\tau)$ and $N_{\|}=\mathbf{b}\left(\mathbf{x}_{0}(\tau)\right) \cdot \mathbf{N}=b^{\alpha}(\tau) N_{\alpha}$, while from (2) we obtain

$$
\Delta N_{\|}(\tau)=\frac{1}{\sqrt{2 k}}\left[b^{\alpha}(\tau) b^{\beta}(\tau) \frac{\partial}{\partial B_{1}^{\alpha \beta}} \log \operatorname{det} B_{1}(\tau)\right]^{\frac{1}{2}}
$$

where $\log \operatorname{det} B_{1}$ is regarded as a function of the entries $B_{1}^{\alpha \beta}$ of the matrix $B_{1}=\left(B_{1}^{\alpha \beta}\right)$.

Let us remark that in toroidal geometry with no poloidal magnetic field we have $\mathbf{b}=b^{\phi} \nabla \phi$, where $\phi$ is the toroidal angle. Hence, $N_{\|}=b^{\phi} N_{\phi}, N_{\phi}$ being the toroidal component of the refractive index, and this relationship transfers to both the central value and the width of the distribution, namely, $N_{\|}(\tau)=b^{\phi}(\tau) N_{\phi}(\tau)$, and $\Delta N_{\|}=b^{\phi}(\tau) \Delta N_{\phi}(\tau)$. The spectrum in $N_{\phi}$ is particularly relevant since in axisymmetric plasmas the equilibrium is independent on the toroidal angle $\phi$ (i.e., $\phi$ is a cyclic coordinate), hence, ray tracing calculations [7] show that $N_{\phi}$ is constant along the rays and so is $\Delta N_{\phi}$. We wonder whether analogous conservation laws can be stated in presence of diffraction effects. The $N_{\phi}$-spectrum can be described by (2), provided that one of the beam coordinates can be identified with $\phi$, e.g., $v^{2}=\phi$. Then, $N_{2}=N_{\phi}$ and its distribution, obtained from (2), exhibits the central value $N_{\phi}(\tau)=N_{2}(\tau)=$ constant for axisymmetric plasmas; furthermore, from the code LHBEAM we find $\Delta N_{\phi}(\tau)=$ constant too. Besides the numerical results, it is possible to show that whenever $v^{\alpha}$ is cyclic, all the momenta of the normalized $N_{\alpha}$ distribution (in particular, $\Delta N_{\alpha}$ ) are constant in $\tau$. This is not evident a priori, since the tails of the distribution correspond to evanescent harmonics that are reflected back changing the shape of the spectrum: our proof shows that these effects occur at higher orders in $1 / \sqrt{k}$. As a consequence of this conservation law, for axisymmetric plasmas with no poloidal magnetic field, the profiles of $N_{\|}(\tau)$ and $\Delta N_{\|}(\tau)$ are determined by $b^{\phi}(\tau)=1 / R(\tau), R$ being the major radius on the reference ray.

Formula (3) has been studied and tested in a two-dimensional slab geometry with Cartesian coordinates $(x, y)$ and with the magnetic field directed along the $y$-axis; in this case the relevant beam cross-section amount to the field lines, with no further degree of freedom. Here are the results. (i) For free-space propagation, we find the expected constant value $\Delta N_{\|}=c /\left(\omega w_{0}\right)$, where $w_{0}$ is the beam width at the waist. (ii) In correspondence of an $x$-dependent electron density profile we find $\Delta N_{\|}=$constant; in this case the magnetic field points along a cyclic variable. (iii) The constant value obtained in (ii) is in agreement with that computed from the numerical Fourier transform of the beam tracing solution. In general we find that the value of $\Delta N_{\|}$is the result of a non trivial combination of the beam width and the curvature of the phase front; an example is given by the case of free-space propagation (i) in which the beam width balances the effect of the phase-front curvature yielding the constant value of the parallel spectral width. 
LHBEAM results. The spectral width (3) has been implemented in the beam tracing code LHBEAM. Nevertheless, the results thus obtained should be considered very preliminary: although the expression (3) has been rigorously derived, the underlying choice of the coordinates $\mathbf{v}=\left(v^{1}, v^{2}\right)$ spanning the beam cross-sections is, at the moment, poorly justified for the reasons discussed above.

On the other hand, our numerical results are well in agreement with the theory. More specifically, the conservation law $\Delta N_{\phi}=$ constant has been clearly confirmed as well as the expected relation between $\Delta N_{\|}$and $\Delta N_{\phi}$ for the case of purely toroidal magnetic field. In addition, upon entering a very large value for the tokamak major radius $R_{0}$, we can reproduce in the code a slab geometry, and in that case we found $\Delta N_{\|}=$constant (cf., point (ii) in the previous section). From another point of view, these results provide a further check of the accuracy of the integration of the beam tracing equations in the LHBEAM code.

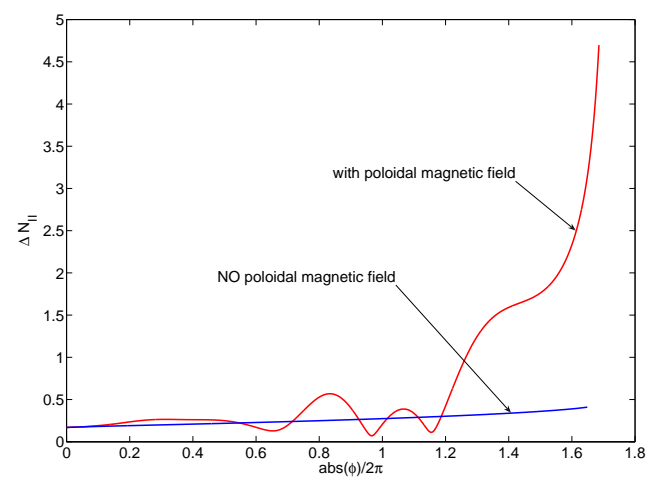

FIGURE 1. The spectral width $\Delta N_{\|}$, Eq. (3) in coordinates $(s, u)$ defined in the text, with and without poloidal magnetic field.

As an example, Fig. 1 shows the spectral width (3) as a function of the toroidal angle $\phi$ (which plays the role of $\tau$ ) with and without the poloidal component of the magnetic field. The following plasma parameters are used: major radius $R_{0}=296 \mathrm{~cm}$, minus radius $a=125 \mathrm{~cm}$, Shafranov shift $\Delta=0$, elongation $\kappa=1$ and toroidal magnetic field $B=3.45 \mathrm{~T}$ on the magnetic axis. The LH beam frequency is $\omega / 2 \pi=3.7 \mathrm{GHz}$. The profile of the electron density $n_{e}$ and the safety factor $q$ are given in the form $n_{e}=\left(n_{e, 0}-n_{e d g}\right)\left(1-\rho^{2}\right)+n_{e d g}$ with $n_{e, 0}=3 \times 10^{13} \mathrm{~cm}^{-3}$ and $n_{e d g}=3 \times 10^{12} \mathrm{~cm}^{-3}$, and $q=1+3 \rho^{2}$ ( $\rho$ is the normalized minor radius). The initial value of parallel refractive index is $N_{\| .0}=1.8$. The beam is launched in equatorial plane, from low-field side with $N_{\vartheta}=0$ and the full electromagnetic dispersion relation is solved.

From Fig. 1 it appears that the effect of the poloidal component of the magnetic field yields a significant broadening of the parallel energy spectrum, yet this result is non conclusive as it depends on the choice of the beam cross-sections as stated above.

\section{COMPARISON OF LHBEAM AND C3PO CODES}

As mentioned at the beginning, LHBEAM solves the beam tracing equations in a tokamak geometry for arbitrary launching conditions and for analytic magnetic equilibria. In 
particular, the plasma dielectric tensor is calculated in the cold plasma limit and in the range of LH frequency approximation $\left(\omega_{c i}^{2} \ll \omega^{2} \ll \omega_{c e}^{2}\right)$ and the code allows us to choose between the full electromagnetic and electrostatic dispersion function.

In order to perform a check of the accuracy of LHBEAM, we compare the trajectory of the central ray of LHBEAM, which obeys the standard ray tracing equations with the result obtained by means of $\mathrm{C} 3 \mathrm{PO}$ code, which is a ray tracing code for an inhomogeneous and anisotropic plasmas with arbitrary axisymmetric magnetic equilibrium and nested flux surfaces $[3,8]$. Parameters employed in this comparison are the same of the parameters used in the previous section along with a second run with $q=$ constant and $N_{\|, 0}=1.5$.

From Figs. 2 the ray trajectories of two codes appear very similar; there is an excellent agreement in the toroidal wave propagation (Fig. 2(b)) whereas small differences appear in the poloidal wave propagation (Fig. 2(a)), in particular, in the last part of the ray trajectory.
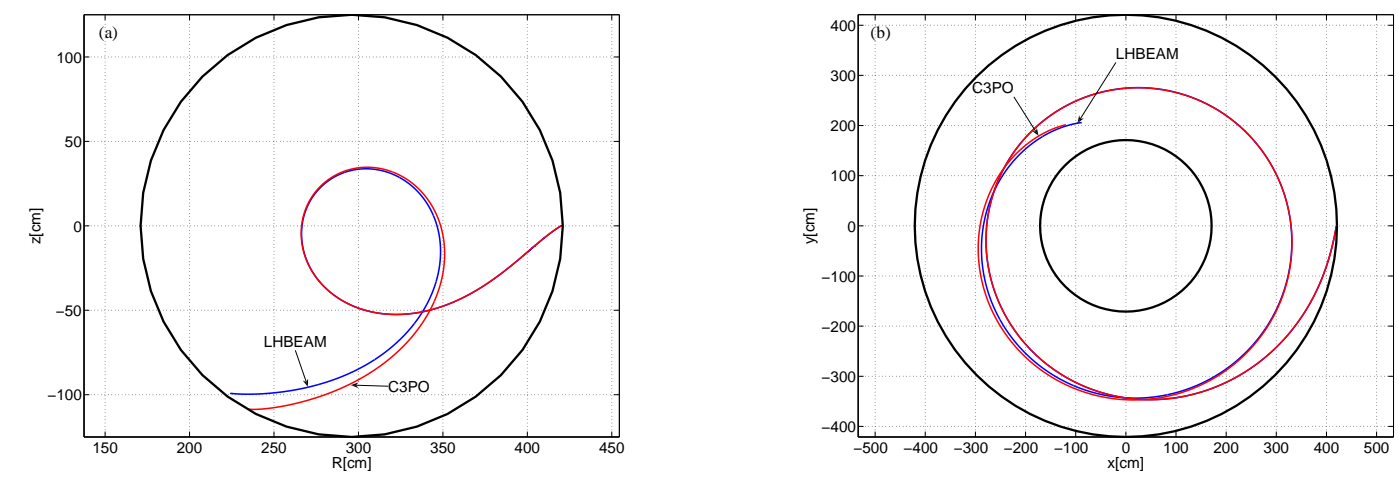

FIGURE 2. The central ray trajectory of LHBEAM with the corresponding ray of C3PO for $N_{\|}=1.8$ and $q=1+3 \rho^{2}$. (a) Poloidal and (b) toroidal wave beam propagation.
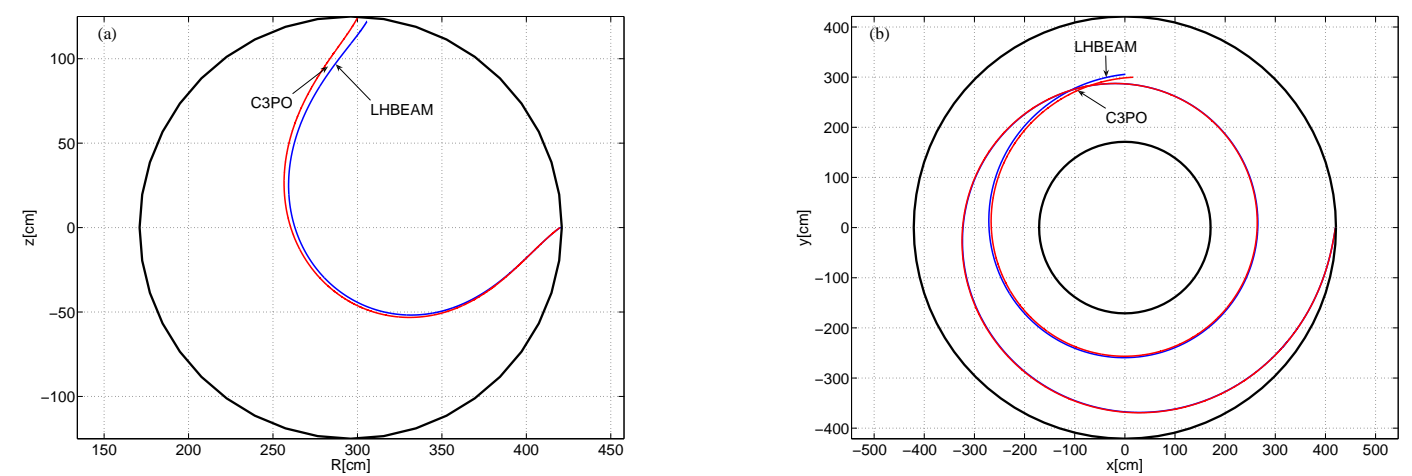

FIGURE 3. The central ray trajectory of LHBEAM with the corresponding ray of C3PO for $N_{\|}=1.5$ and $q=4$. (a) Poloidal and (b) toroidal wave beam propagation.

In order to try to understand the cause of these differences we consider a second run with $q=4$ and $N_{\|, 0}=1.5$, which is shown in Figs. 3 . The specific choice of these parameters is aimed to simplify the poloidal magnetic field profile for both codes (see 

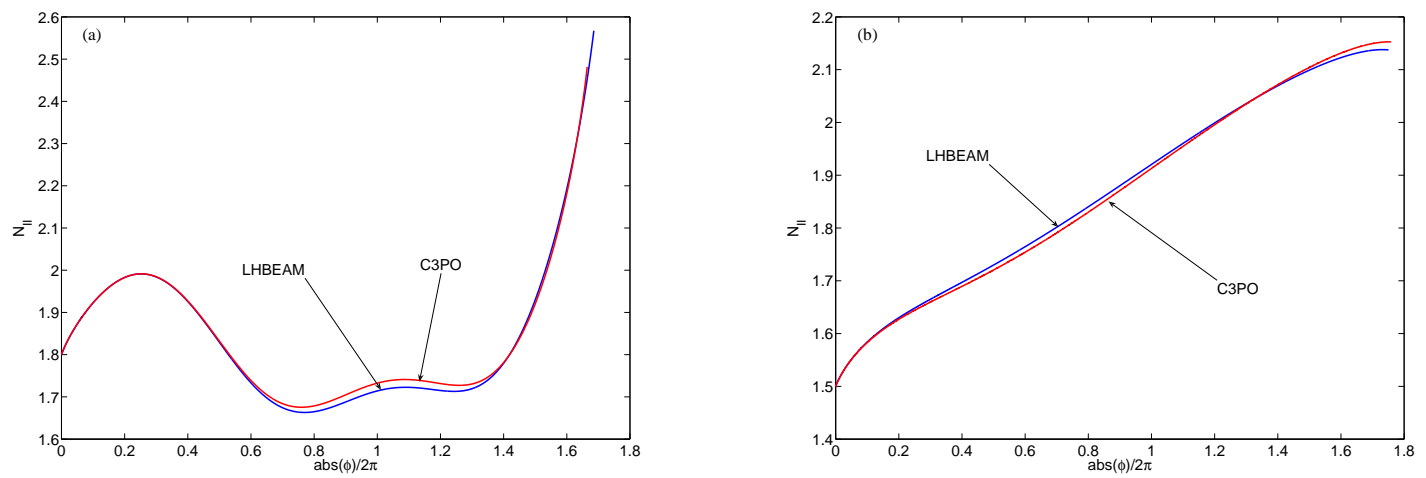

FIGURE 4. The parallel refractive index as a function of toroidal angle $\phi$ for LHBEAM and C3PO. (a) $N_{\|}=1.8$ and $q=1+4 \rho^{2}$; (b) $N_{\|}=1.5$ and $q=4$.

Refs. [5] and [8] for details, respectively). From poloidal wave propagation (Fig. 3(a)) one sees that the small differences of ray trajectories are still there but smaller than before; on the other hand, from the toroidal ray propagation (Fig. 3(b)), the agreement remains very good. A comparison between the evolution of the parallel refractive index $\left(N_{\|}\right)$as a function of the toroidal angle $\phi$ is plotted in Figs. 4 for both cases and confirms the good agreement between two codes. Summarizing, for the cases considered, the comparison of LHBEAM and C3PO is satisfactory despite the fact that there are small discrepancies due to, seemingly, the different procedures adopted in the two codes for the calculation of the equilibrium.

\section{ACKNOWLEDGMENTS}

Authors wish to thank R. Bilato and M. Brambilla for useful discussions. This work, supported by the European Communities under the contract of Association between EURATOM/FOM, was carried out within the framework of the European Fusion Programme and EFDA. The views and opinions expressed herein do not necessarily reflect those of the European Commission. The work of O.M. has been supported by the Foundation BLANCEFLOR Boncompagni-Ludovisi.

\section{REFERENCES}

1. G. V. Pereverzev, Nucl. Fusion 32, 1091 (1992).

2. P. T. Bonoli, et al., in 21st IAEA Conf., Chengdu, China (2006).

3. Y. Peysson, J. Decker and V. Basiuk, 34th EPS Conf. on Plasma Phys. (Warsaw) Vol 31F (ECA) P4.164 (2007).

4. G. V. Pereverzev, Reviews of Plasma Physics 19, 1 (1996); Phys. Plasmas 5, 3529 (1998).

5. N. Bertelli, G. V. Pereverzev and E. Poli, 34th EPS Conf. on Plasma Phys. (Warsaw) Vol 31F (ECA) P5.051 (2007); N. Bertelli, A beam tracing code for the description of LH wave propagation in a tokamak plasma, Ph.D thesis, University of Pavia (2007). ISBN 978-88-95767-04-8.

6. E. Poli, A. G. Peeters and G. V. Pereverzev, Comput. Phys. Commun. 136, 90 (2001).

7. M. Brambilla and A. Cardinali, Plasma Physics 24, 1187 (1982).

8. Y. Peysson and J. Decker, C3PO, a ray tracing code for arbitrary axisymmetric magnetic equilibrium, EURATOM-CEA Internal Report (2008). 